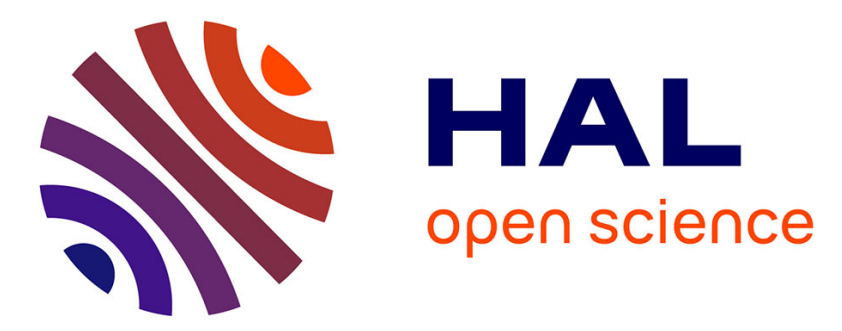

\title{
Adaptation des TIC dans un contexte pédagogique pour des élèves en situation de multi handicap.
}

\author{
Amélie Roche, Véronique Lespinet-Najib, Valérie Demanes, Isabelle
}

Fermingier, Jean-Marc André

\section{- To cite this version:}

Amélie Roche, Véronique Lespinet-Najib, Valérie Demanes, Isabelle Fermingier, Jean-Marc André. Adaptation des TIC dans un contexte pédagogique pour des élèves en situation de multi handicap.. Terminal. Technologie de l'information, culture \& société, 2014, 10.4000/terminal.673 . hal01702600

\section{HAL Id: hal-01702600 \\ https://hal.science/hal-01702600}

Submitted on 6 Feb 2018

HAL is a multi-disciplinary open access archive for the deposit and dissemination of scientific research documents, whether they are published or not. The documents may come from teaching and research institutions in France or abroad, or from public or private research centers.
L'archive ouverte pluridisciplinaire HAL, est destinée au dépôt et à la diffusion de documents scientifiques de niveau recherche, publiés ou non, émanant des établissements d'enseignement et de recherche français ou étrangers, des laboratoires publics ou privés. 


\title{
Terminal
}

Technologie de l'information, culture \& société

116 | 2015 :

TIC \& handicap

TIC \& Handicap

TIC \& Apprentissage

\section{Adaptation des TIC dans un contexte pédagogique pour des élèves en situation de multi- handicap}

\author{
Amélie Roche, Véronique Lespinet-Najib, Valérie Demanes, Isabelle \\ Fermingier et JeAn-Marc André
}

\section{Résumés}

Français English

Ce travail présente une méthode de conception systémique fondée sur le design universel et la conception centrée utilisateurs (CCU), permettant de proposer des outils et services qui répondent aux besoins et attentes des populations dites fragiles. Cette méthode a été testée dans un contexte pédagogique auprès d'enfants en situation de multihandicap. L'objectif est de fournir un outil d'aide pour l'apprentissage de la lecture. Selon la démarche proposée et afin d'offrir une solution adaptée, nous avons mis en place des phases successives et itératives lors desquelles nous mettons en relation les profils utilisateurs (en termes de capacités cognitives, sensorielles et motrices, de besoins et de points forts des enfants) ; les situations d'actions caractéristiques (exercices issus de la méthode d'acquisition de la lecture mise en place par l'enseignante) et le contexte. Nous avons ainsi conçu un outil numérique permettant aux enfants de réaliser des exercices de lecture avec plus d'autonomie. Différents prototypes ont été testés selon la démarche de CCU. Les résultats sont concluants : l'expérience utilisateurs des élèves et l'acceptabilité de l'outil sont très satisfaisantes. Celui-ci répond aux besoins et attentes des élèves ainsi qu'à ceux de l'enseignant. Ces premiers travaux montrent que notre méthode de conception systémique semble être pertinente. 
This work presents a systemic design method, based on universal design and user-centred conception (UCC), aiming to provide tools and services that truly answers to needs and expectations of "fragile" populations, as people with disabilities. This method has been tested in an educational context with pupils with multiple disabilities. The objective here is to provide a tool for helping children in learning to read. According to the proposed approach, we have performed successive and iterative phases during which we have crossed the following information in order to provide a suitable solution : 1) pupils profiles (in terms of cognitive, sensory and physical abilities ; needs and strengths of pupils) ; 2) characteristic situations of uses (task that pupils perform in educational context) and the cognitive, sensory and physical requirement to perform each of them ; 3) context (environment, actors, materials, etc.). We have designed a tool allowing children to make reading exercises with increased autonomy. Different prototypes were tested according to the users-centred design approach. The results of this work are conclusive: user experience of pupils and acceptability of this tool are very satisfying. This tool answers to pupils' needs and expectations, and it also answers to those of the teacher. This first experiment shows that our systemic design method seems to be relevant.

\section{Entrées d'index}

Mots-clés : TIC, méthode de conception systémique, multi-handicap, outil d'aide pédagogique, conception universelle, conception centrée utilisateurs

Keywords : ICT, systemic design method, multiple disabilities, pedagogical aid tool, universal design, user centred design

\section{Texte intégral}

\section{Introduction}

En France, les établissements régionaux d'enseignement adapté (EREA) sont des établissements publics locaux d'enseignement (EPLE) du second degré. Leur mission est de prendre en charge des enfants et des adolescents en grandes difficultés scolaire et sociale ou présentant un handicap, et de leur permettre de suivre une scolarité adaptée. Une des principales complexités réside dans la diversité de ces élèves. Ils peuvent, dans certains cas, présenter des troubles moteurs et sensoriels, mais aussi des troubles associés incluant certaines déficiences cognitives, troubles attentionnels, problèmes de mémorisation, etc. [Cremadeills et al. 2002]. On parle alors de situation de multihandicap [Cremadeills et al. 2002].

Les rythmes d'apprentissage sont aussi très hétérogènes et peuvent varier très fortement d'un élève à l'autre, mais ces derniers nécessitent tous beaucoup d'attention de la part de l'enseignante. L'intégration des technologies de l'information et de la communication (TIC) au sein de tels établissements constituerait une aide non négligeable pour le soutien à la mise en place de méthodes pédagogiques adaptées à chaque enfant. Bien que ces dernières décennies aient connu une évolution considérable en termes de développement des TIC, les solutions proposées actuellement sont souvent peu, voire pas, adaptées aux particularités des situations de multi-handicap. Elles le sont encore moins dans le cadre de systèmes éducatifs spécifiques où les élèves présentent de plus en plus de déficiences, ce qui nécessite un vrai travail de recherche en pédagogie.

Dans le domaine de la conception technologique, un des concepts clés est celui de la conception centrée utilisateurs (CCU), qui place l'utilisateur final et la tâche qu'il doit effectuer au centre de la démarche de conception [Cremadeills et al. 2002]. La CCU se décompose en différentes phases interdépendantes s'effectuant de manière itérative 
(voir Figure 1). Ces phases sont réalisées en collaboration avec les utilisateurs finaux qui sont impliqués dans l'ensemble du processus de conception.

Figure 1. Interdépendance des différentes étapes de la CCU

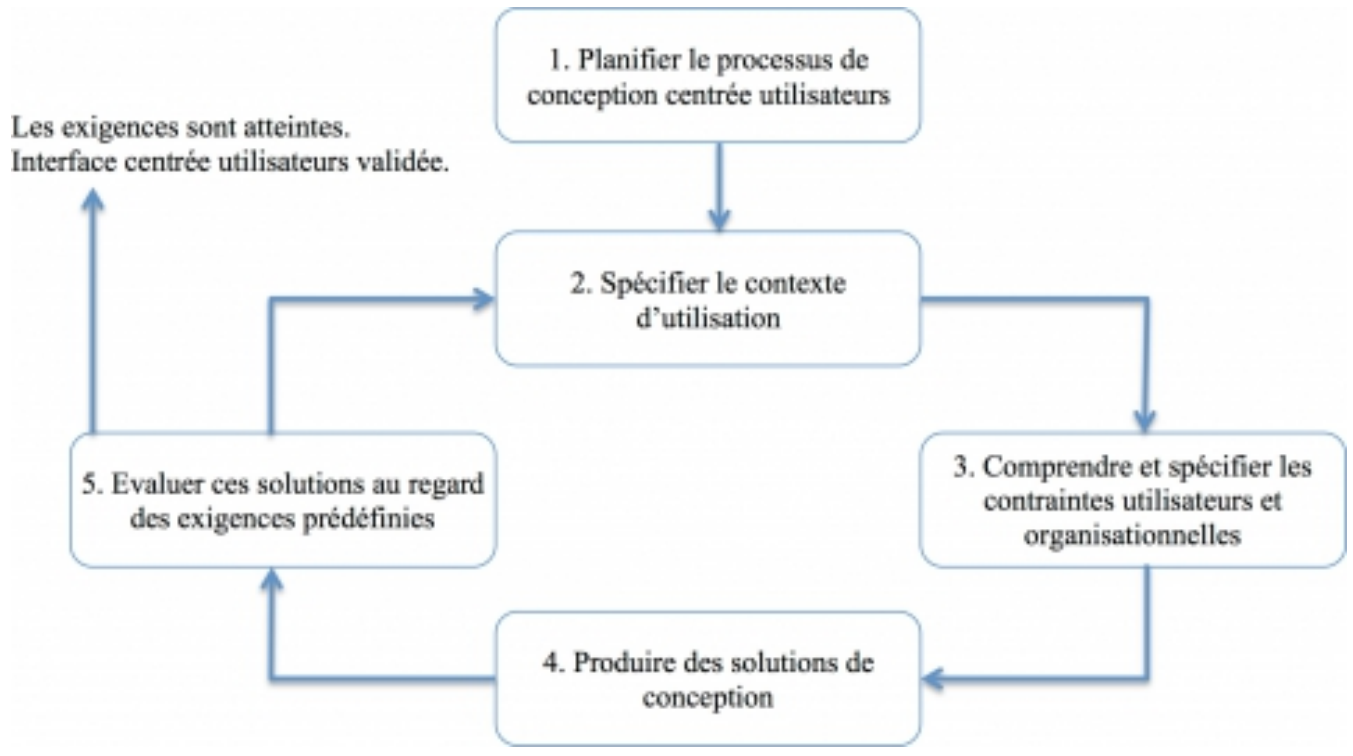

Il existe de nombreuses méthodes de CCU (tests utilisateurs, entretiens, etc.), [Nielsen 1994 ; ISO16982 2002]. Cependant, elles ne prennent pas en compte les particularités motrices, sensorielles et cognitives des personnes en situation de multihandicap. Par exemple, il apparaît complexe de proposer la méthode du questionnaire à une personne souffrant de déficiences cognitives. La CCU vise à améliorer l'utilisabilité d'un produit ou service, définie comme " le degré selon lequel un produit peut être utilisé par des utilisateurs identifiés pour atteindre des buts définis avec efficacité, efficience et satisfaction, dans un contexte d'utilisation spécifié » [ISO13407 1999].

Comme le précise Choi (2005), la CCU repose sur les concepts de responsabilité et d'utilisabilité, elle va ainsi concevoir pour le plus grand nombre (la majorité). Dillon \& Morris (1999) insistent sur le fait que l'utilisabilité est un déterminant nécessaire, mais non suffisant pour assurer un usage effectif et accordent de l'importance à l'appréciation subjective de la technologie par les utilisateurs. Ils proposent un modèle de l'intention d'usage (et donc de l'acceptabilité) fondé sur trois facteurs :

- L'utilité (fonctionnalité du système en termes de capacités techniques permettant de réaliser la tâche de l'utilisateur) ;

- $\quad$ La perception (évaluation subjective du système par l'utilisateur) ;

- L'utilisabilité (aspect opérationnel du système, performance que l'utilisateur peut atteindre avec la technologie).

Il apparaît donc primordial de coupler les mesures objectives et subjectives pour assurer l'acceptabilité d'un système.

Une approche de conception encore peu développée en France se distingue toutefois des autres méthodes : la conception pour tous, qui tend à concevoir des produits et des services accessibles pour tous (Design for all foundation). Cette démarche de conception centrée sur les utilisateurs et leurs besoins préconise la prise en compte des besoins de tous, quels que soit leur genre, leur âge ou leurs capacités cognitives, sensorielles ou motrices. Ainsi, comme le souligne Choi (2005), la conception pour tous repose sur le concept de diversité et d'inclusion. La démarche est simple, il s'agit de prendre en compte au préalable les contraintes de la minorité et ensuite d'inclure la majorité. La conception pour tous repose sur sept principes fondamentaux (Center for 
Universel Design) : une utilisation équitable pour tous ; une flexibilité et une souplesse d'usage ; une utilisation simple et intuitive ; des informations perceptibles immédiatement ; une tolérance à l'erreur ; un faible niveau d'effort physique ; une dimension et une utilisation de l'espace pour une bonne approche et utilisation.

Mace et al. (1991) définit ce concept comme "visant à concevoir, développer et mettre sur le marché des produits, des services, des systèmes ou des environnements courants qui soient accessibles et utilisables par le plus large éventail possible d'usagers ».

Le design universel ne s'adresse pas uniquement aux personnes en situation de handicap, mais à l'ensemble des individus, il envisage l'accessibilité (Interface hommemachine (IHM), transports, etc.) comme une source d'innovation technologique. Il replace au premier plan la prise en compte des facteurs humains dont chacun peut bénéficier (simplicité, confort, etc.) [Lespinet-Najib et al. 2013]. C'est en se reposant sur cette méthode qu'il nous semble pertinent d'intégrer les caractéristiques des personnes présentant des contraintes maximales (motrices, sensorielles ou cognitives) dans la démarche de conception systémique que nous proposons. En effet, c'est en prenant en compte la diversité des individus [Choi 2005] dans les démarches de conception que l'on pourra fournir des produits qui répondent aux besoins de tous, y compris des minorités. L'idée étant de concevoir des produits dont chacun peut bénéficier, sans nécessité d'ajustement particulier et adaptés au contexte d'usage.

Afin de répondre à un besoin réel identifié par les acteurs de terrain, l'objectif de ce travail est double. Premièrement, formaliser la méthode de conception systémique de IHM permettant d'offrir des outils et des services qui répondent réellement aux besoins et aux attentes des personnes en situation de multi-handicap ; deuxièmement, vérifier la faisabilité de l'intégration d'un outil numérique dans un contexte pédagogique auprès d'élèves en situation de multi-handicap.

\section{Démarche méthodologique}

\section{Le terrain}

Le terrain d'intervention de notre démarche est un établissement régional d'enseignement adapté (EREA) et Lycée d'enseignement adapté (LEA) localisé dans la région Aquitaine, l'EREA/LEA « La Plaine ». Cet établissement présente certaines spécificités. Il est composé de quatre structures : le primaire, le collège, le collège Segpa (Section d'Enseignement général et professionnel adapté) et le lycée professionnel. L'établissement est localisé à proximité d'un IEM (Institut d'Éducation motrice), établissement proposant diverses actions sous les angles éducatifs, mais aussi médicaux. Les enfants qui arrivent à l' EREA pour le primaire et le collège sont orientés par les MDPH (Maisons départementales des personnes handicapées) sur un motif de base lié à un handicap moteur. Cependant, il est constaté à l'arrivée des enfants qu'ils présentent en plus des troubles cognitifs et sensoriels : difficulté de mémorisation, de communication, problèmes attentionnels, etc. Ces élèves ne peuvent pas suivre un rythme ordinaire, ni même adapté tel que le proposent les CLIS (Classes pour l'inclusion scolaire) et les ULIS (Unités pédagogiques d'intégration), dont l'objectif est d'aider les enfants en difficultés scolaires liées à leur handicap psychologique, moteur ou sensoriel, dans les écoles primaires et secondaires. Les enseignants doivent faire preuve d'une grande adaptabilité et mettent en place des méthodes pédagogiques 
innovantes pour répondre aux besoins spécifiques de chaque enfant. Cette étude a été menée en collaboration avec cet EREA, plus particulièrement auprès d'une classe de treize enfants, âgés de treize à dix-neuf ans.

\section{Mise en pratique de la méthodologie innovante}

\section{Le concept}

La méthode que nous proposons se veut systémique dans le sens où elle considère la problématique dans sa globalité. L'ensemble des éléments est pris en compte. En nous reposant sur la démarche de conception pour tous, nous intégrons les spécificités motrices, sensorielles ou cognitives des utilisateurs finaux ainsi que les éléments contextuels, tels que les contraintes organisationnelles. Comme le souligne Belio (2012), la problématique du handicap est à prendre dans sa globalité. L'ensemble de l'environnement (matériel ou humain) joue un rôle essentiel dans le succès de l'intégration d'un système numérique. La démarche de conception systémique se compose de trois principales phases (voir Figure 2):

- Définition et application de grilles d'analyse ;

- Alignement des éléments récupérés via les grilles d'analyse ;

- $\quad$ Conception et validation de l'outil.

Figure 2. Étapes successives et itératives de la méthode de conception systémique

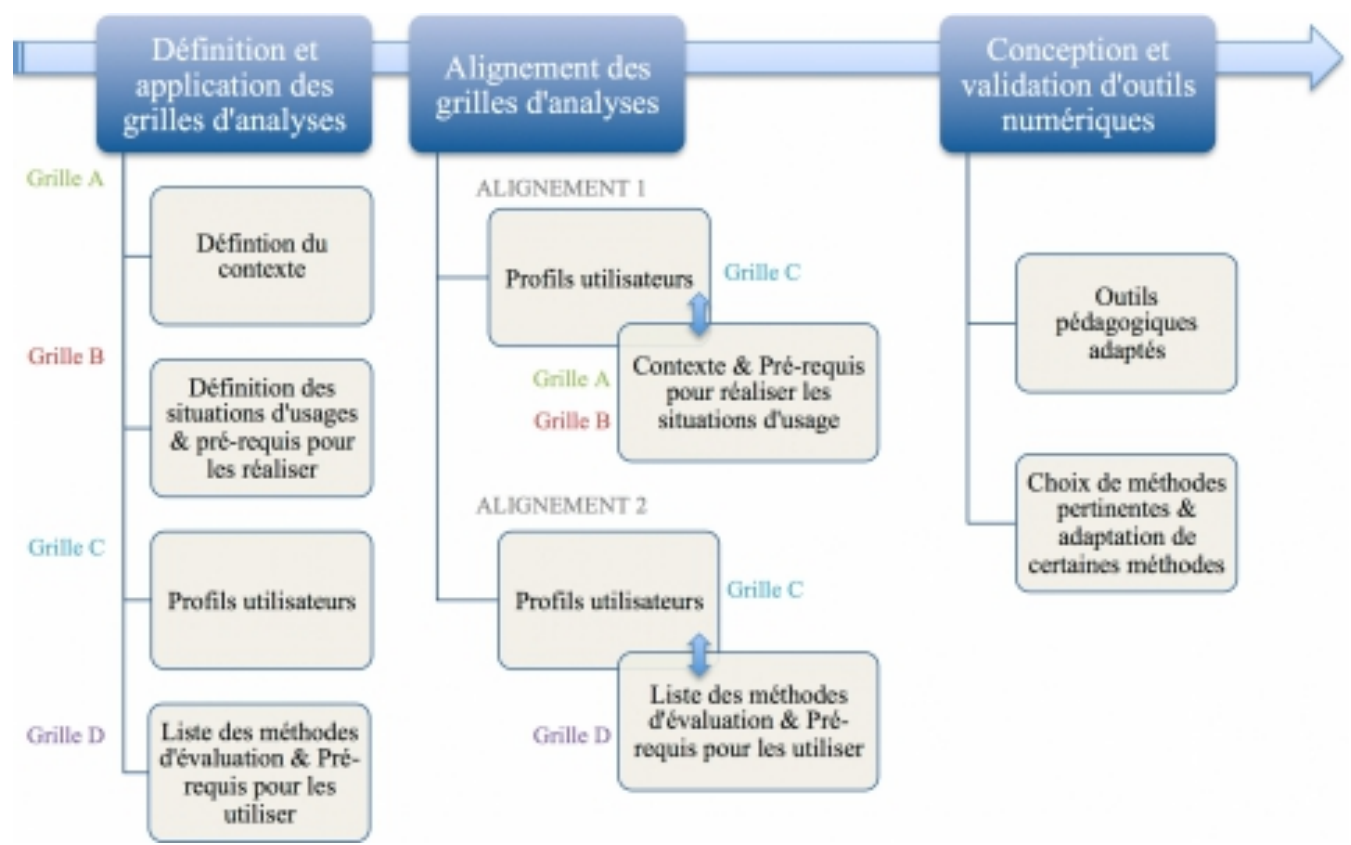

Nous avons suivi chacune de ces étapes pour concevoir l'outil d'aide pédagogique destiné aux élèves en situation de handicap, elles sont explicitées dans la suite du document.

\section{Définition et application des grilles d'analyse}

La première étape consiste en la définition de grilles d'analyse des éléments suivants : le contexte, les situations d'actions caractéristiques, les profils utilisateurs et 
les méthodes d'évaluation de l'utilisabilité d'une IHM. réaliser

\section{Grille A : Le contexte}

Il s'agit d'identifier les éléments qui composent l'environnement des utilisateurs et avec lesquels ils interagissent : acteurs (internes, externes, etc.), transport, etc. Nous avons mené des entretiens semi-directifs auprès du personnel de l'EREA (enseignants, auxiliaire de vie (AVS), du personnel administratif, logistique, soignant) et des séances d'observation en classe. Les éléments identifiés sont représentés sous la forme de carte conceptuelle élaborée avec le logiciel XMind (Fig. 3). Une carte conceptuelle représente un savoir organisé, elle facilite la lecture et l'appropriation des informations [Novak et Cañas, 2008].

Figure 3 : Représentation des éléments qui composent l'environnement (logiciel XMind)
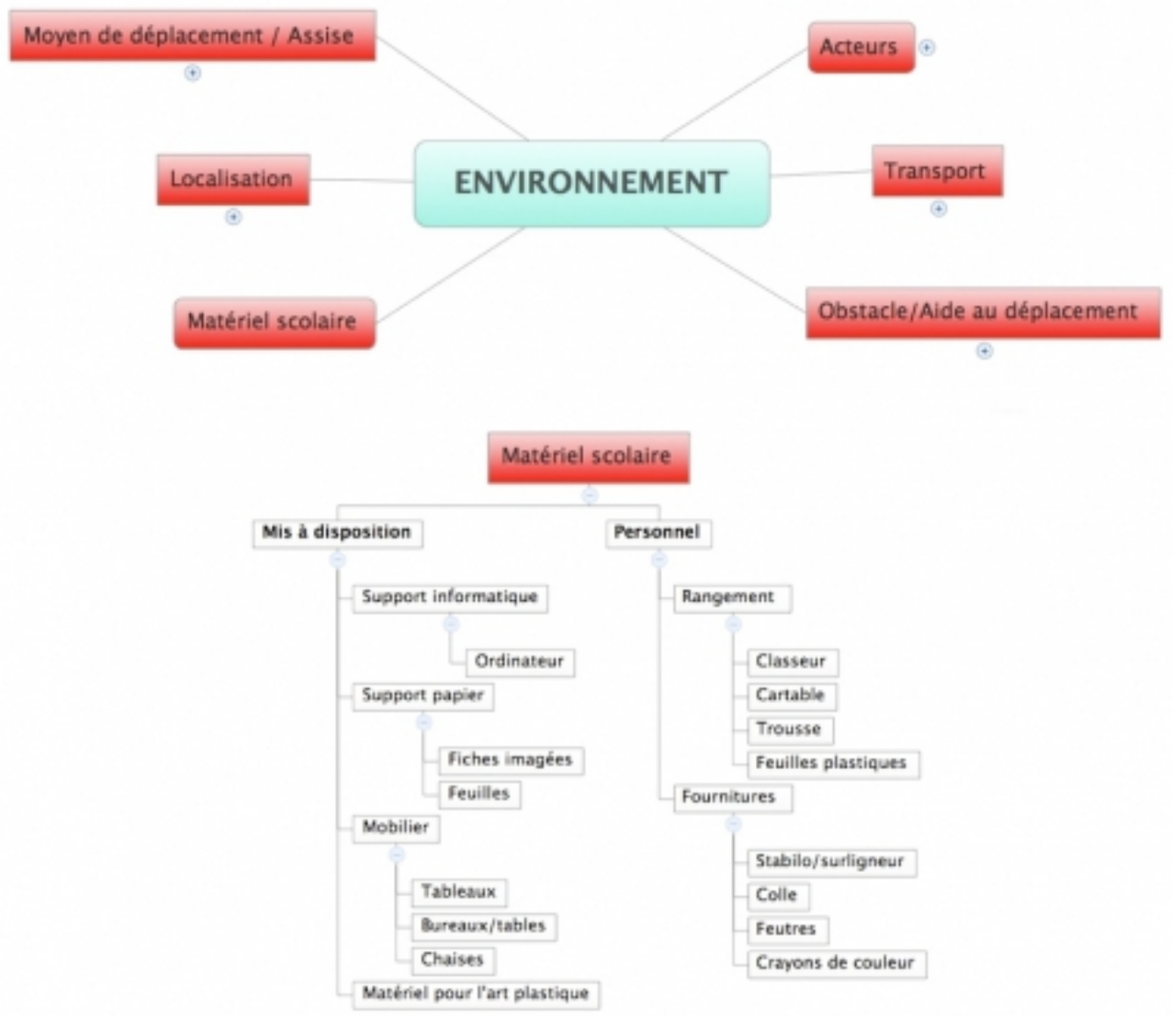

\section{Grille B : Les situations d'actions caractéristiques}

Il s'agit tout d'abord d'identifier l'ensemble des situations d'actions caractéristiques (i.e. des tâches que réalisent les enfants) ; pour ensuite définir des prérequis cognitifs, sensoriels et moteurs nécessaires permettant de réaliser ces tâches (mémoire de travail, manipulation d'objet, etc.). Afin de cadrer le projet, nous avons décidé avec les intervenants de l'EREA de nous focaliser d'abord sur les cours de français, et principalement sur l'activité de lecture. Nous avons déterminé l'ensemble des tâches que réalisent les élèves et, pour chacune d'entre elles, son objectif et les prérequis nécessaires pour les réaliser (Fig. 4).

Figure 4 : Exemple d'une situation d'action caractéristique et des prérequis pour la 


\section{APPRENTISSAGE DES SONS ASSOCIES À UNE GRAPHIE}

Principe : Le son est la base à acquérir, l'image est un médiateur d'aide pour accéder à des graphies dont les sonorités ne sont pas stables.

\section{Prérequis nécessaires}

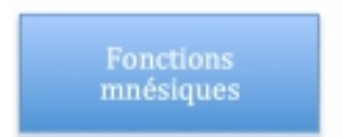

•Visuelle

-Phonologique

-Sémantique

-Etc.
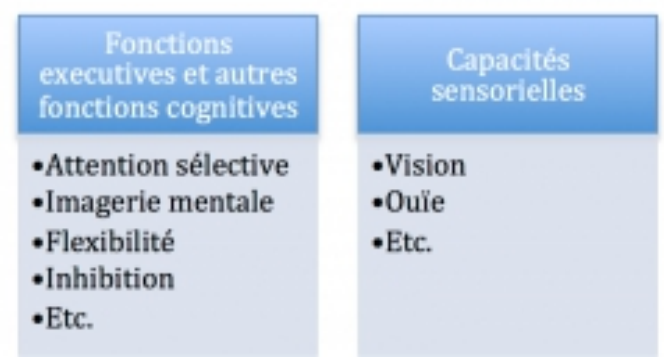

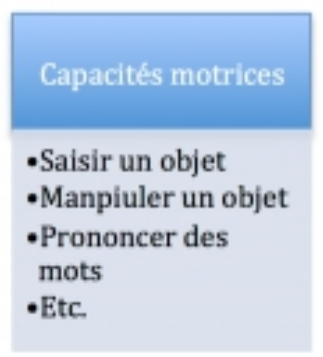

\section{Grille C : Les profils utilisateurs}

Nous avons ensuite décomposé les profils des élèves selon trois catégories : leurs déficiences (cognitives, sensorielles et motrices), leurs besoins et leurs points forts. Ces éléments ont été déterminés via des entretiens auprès des enseignants, des AVS et du personnel soignant et via des séances d'observation en classe. Dans le cadre de cette recherche préliminaire, nous travaillons avec trois élèves (des filles) qui ont des profils assez similaires. Elles présentent de fortes contraintes cognitives, sensorielles et motrices. En effet, comme précisé préalablement, il s'agit de partir des situations ayant le plus de contraintes. La figure 5 donne une brève illustration des éléments recensés.

\section{Figure 5 : Profils des trois élèves en termes de déficiences, besoins et points forts}

\section{Elève 1:}

Déficiences
-Dyspraxie manuelle et
visio-spatiale.
-Dysphasie (difficulté dans
la compréhension et la
production du langage,
etc.).
- Troubles mnésiques
divers dont celui de la
mémoire de travail.
- Etc.

Elèves 2 et 3 :

\begin{tabular}{l} 
Déficiences \\
\hline -Dysphasie : difficulté à \\
produire/exprimer et à \\
comprendre le langage \\
oral. \\
-Mémoire sémantique : \\
difficulté à comprendre ou \\
donner un sens aux mots. \\
-Difficulté d'appréhension \\
de l'abstraction. \\
-Etc.
\end{tabular}
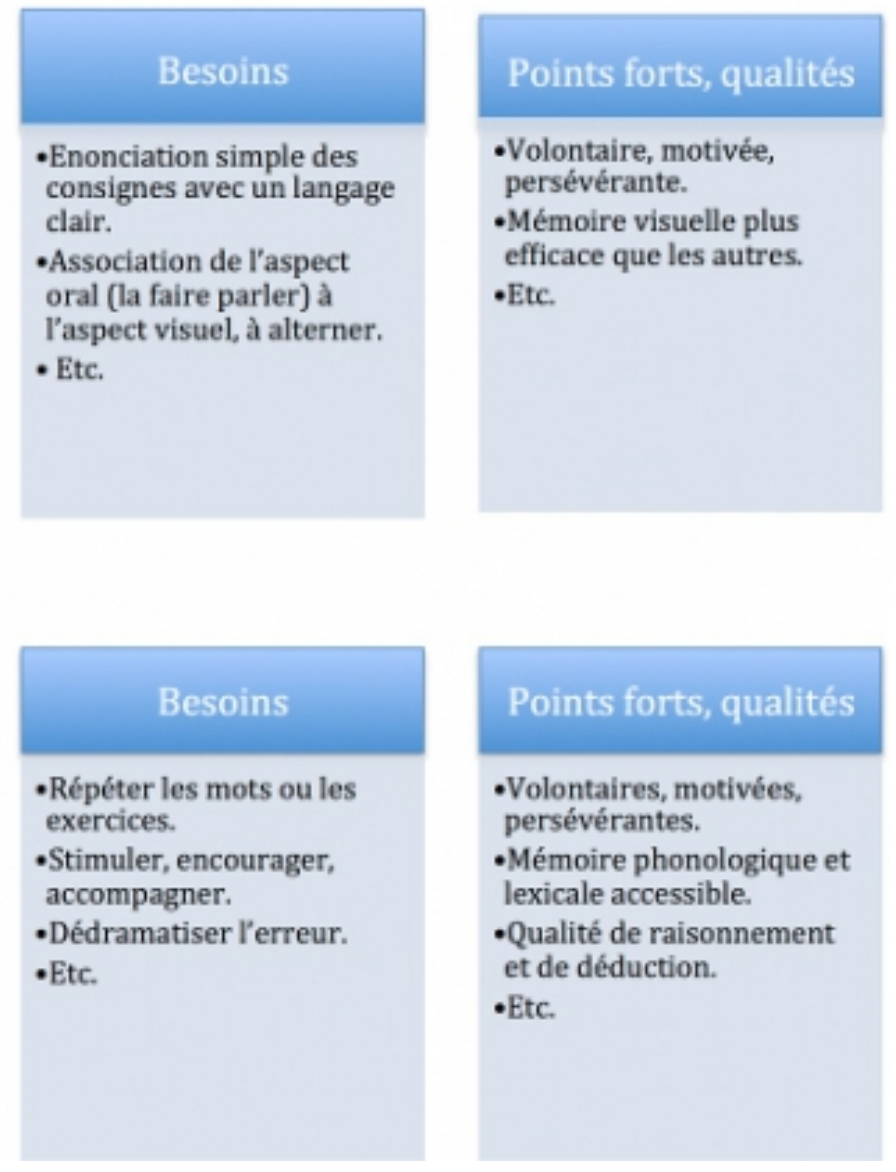

\section{Points forts, qualités}

-Volontaires, motivées, persévérantes.

-Mémoire phonologique et lexicale accessible.

-Qualité de raisonnement et de déduction.

-Etc. 


\section{Figure 6 : Description de trois méthodes d'utilisabilité DESCRIPTION}

L'utilisateur interagit avec une interface. Cette interaction peut être libre (le sujet n'aura pas d'objectifs précis) ou scénarisée (le sujet doit réaliser une tâche précise).

\section{PREREQUIS}

Capacités attentionnelles (sélective et partagée); de mémorisation à court terme (flexibilité, mise à jour, inhibition, etc.) et long terme ; fonctions exćcutives (planification, compréhension,

Capacités attentionnelles (sélective et partagée) ; de mémorisation à court terme et long terme; fonctions exécutives (planification, compréhension, flexibilité mentale, etc.) ; de communication, etc.

\section{Alignement des grilles d'analyses}

Á partir des données définies précédemment, il s'agit de mettre en adéquation :

1. Les profils des utilisateurs (Grille C), le contexte (Grille A) et les situations d'usages caractéristiques (Grille B) afin d'identifier les tâches possibles et proposer des solutions, qu'elles soient numériques ou non.

2. Les profils des utilisateurs (Grille C) et les méthodes d'évaluation de l'utilisabilité des IHM (Grille D) afin de déterminer les méthodes possibles, et si besoin les adaptations nécessaires.

Alignement 1 : profils utilisateurs (Grille C) et situations d'actions caractéristiques et contexte (Grille A et B)

Choix des situations d'actions caractéristiques

Quatre exercices d'introduction à la lecture ont été identifiés :

- $\quad$ Exercice $\mathrm{n}^{\circ} 1$ : lecture d'image

- $\quad$ Exercice $\mathrm{n}^{\circ} 2$ : association mots-image

- $\quad$ Exercice $\mathrm{n}^{\circ} 3$ : les mots d'extras

- $\quad$ Exercice $n^{\circ} 4$ : lecture de mots et recherche sur Internet

Nous avons décidé de développer un logiciel d'aide pédagogique et d'utiliser la tablette tactile comme support. En effet, cette dernière répond aux attentes et aux 
capacités des trois élèves : elles ont la dextérité nécessaire pour interagir avec la tablette et elles ont l'habitude de manipuler cet outil au sein de leur foyer. En outre, les élèves ont une représentation positive de cet outil et se l'approprient facilement, ce qui constitue un élément majeur pour son acceptabilité [Dillon \& Morris 1999]. Cet outil répond aux besoins et aux attentes que peut avoir un enseignant dans le cadre d'une salle de classe : facilement maniable, transférable d'un élève à un autre, peut être emporté à l'extérieur lors de sorties pédagogiques, etc.

Alignement 2 : profils utilisateurs (Grille C) et méthodes d'évaluation de l'utilisabilité (Grille D)

Deux principales méthodes ont été ciblées : l'observation et le scénario d'usage, dont l'utilisation a été adaptée au contexte particulier de l'EREA. Par exemple, pour le déroulement des scénarios d'usage, nous allons veiller à ne pas laisser de choix possible dans la navigation pour ne pas risquer de perdre l'élève. De plus, nous apporterons pour les deux méthodes une attention particulière à l'accompagnement de l'élève pour le rassurer et faire en sorte qu'il ne se considère pas en situation d'évaluation.

\section{Conception de l'outil et résultats}

Á partir des éléments issus des différents alignements, nous avons conçu un outil d'aide pédagogique selon une démarche itérative. Cet outil reprend les exercices identifiés : la lecture d'image, l'association mot-image, les mots d'extras, la lecture de mots.

Lors de la conception, nous avons veillé à ce que notre outil réponde aux critères heuristiques utilisés en ergonomie cognitive [Bastien \& Scapin 1992 ; Nielsen 1994], les critères d'incitation (les étapes se succèdent les unes aux autres de manière linéaire pour ne pas laisser de choix de navigation aux élèves) ; de distinction entre items (alternances de couleurs et contrastes assez importants) ; ou de lisibilité (les images et les mots sont de taille assez grande pour en faciliter la distinction et la lecture); etc. De plus, la conception de notre outil repose aussi sur les sept principes du design universel. En parallèle, nous avons développé un site Web permettant à l'enseignante de paramétrer l'ensemble des exercices : elle peut choisir les images, leur associer un son, définir les exercices en fonction des niveaux, etc.

Nous avons élaboré deux prototypes qui ont été évalués auprès des enfants avec les méthodes d'observation et de scénario d'usage. L'évaluation de l'outil s'est déroulée en situation réelle, au sein d'une classe de cours en présence de l'enseignante pour conserver les conditions normales et ne pas déstabiliser les élèves. L'objectif était de les laisser interagir avec l'outil, en les accompagnant au besoin. Des améliorations ont été apportées au fur et à mesure des tests. Par exemple, ceux réalisés sur le premier prototype ont permis d'identifier des modifications à apporter pour faciliter la lisibilité : accentuation du contraste, changement de police, alternance des couleurs, etc. Les élèves ayant d'importantes déficiences visuelles, l'alternance des couleurs et le contraste proposés initialement n'étaient pas suffisants.

Des fonctionnalités ont également été ajoutées pour accompagner davantage les élèves dans la réalisation des exercices : un retour sonore est accessible sur l'ensemble des images et des mots permettant à l'élève de se corriger lors de leur lecture, les élèves ont la possibilité de surligner de différentes couleurs les syllabes pour faciliter la lecture, etc. Les tests sur le second prototype ont mis en évidence le besoin d'améliorer d'une part l'enchaînement des exercices en fonction des niveaux, mais également l'architecture de l'information utilisée pour le paramétrage réalisé par l'institutrice, et 
par conséquent l'affichage sur les écrans tactiles à destinations des élèves. Les résultats du dernier prototype (Fig. 6) sont satisfaisants en terme d'utilisabilité. Les élèves ont réussi à manipuler l'outil sans difficulté et faire les exercices sans aide extérieure : l'IHM s'avère intuitive, avec une navigation guidée et de nombreux feedbacks ; l'utilisation de la tablette n'a pas posé de problème particulier. Les élèves ont ressenti de la satisfaction lors de l'interaction et leur expérience utilisateur est très positive (sourires, commentaires positifs, etc.) : l'acceptabilité par les élèves est satisfaisante.

Figure 7 : Illustrations du système d'aide pédagogique

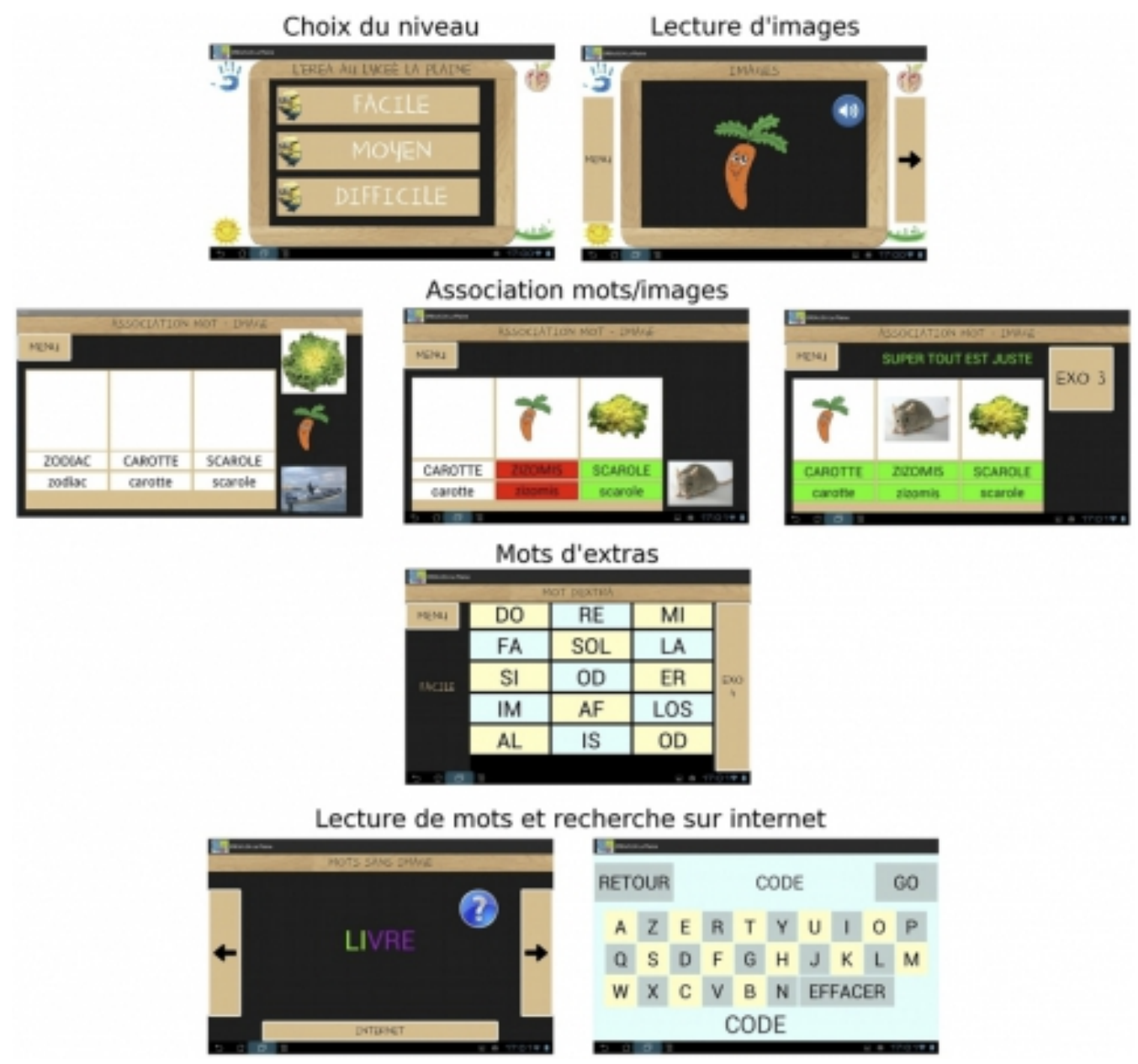

\section{Discussion}

L'objectif de cette étude était d'une part de valider la méthode de conception systémique que nous proposons permettant de prendre en compte les spécificités cognitives, sensorielles et motrices des enfants en situation de multi-handicap, mais également de vérifier la faisabilité de l'intégration d'un outil numérique dans un contexte pédagogique auprès de ces enfants. Les premiers résultats sont concluants puisque les élèves et l'enseignante se sont bien appropriés l'outil ; il répond aux besoins et aux attentes de ces deux profils d'utilisateurs. La méthode systémique proposée, bien qu'elle doive être testée auprès d'autres personnes, semble être pertinente dans le cadre de cette première expérience. Le processus de conception itératif a permis d'adapter en permanence l'outil aux besoins. En suivant le modèle de Dillon \& Morris (1999), nous avons fait en sorte que les conditions nécessaires pour une bonne acceptation de l'outil d'aide pédagogique par les enfants soient remplies : un système utile, utilisable et bien 
perçu. Il apporte une réelle utilité pédagogique : les élèves peuvent réaliser les exercices d'apprentissage de la lecture en autonomie. L'informatisation des sessions d'exercices permet à chaque élève de travailler à son rythme : celui-ci a le temps nécessaire pour prononcer lui-même le mot sans que les plus rapides ne le prononcent avant ; l'aide n'est affichée que sur demande de l'élève ; il a un retour pour lui indiquer si les réponses sont correctes ou non, ce qui lui permet de réaliser l'exercice sans attendre que l'enseignante vienne vérifier, etc. Ainsi l'élève retrouve de l'autonomie dans son apprentissage et est plus actif lorsqu'il réalise les exercices. De plus, l'enseignante peut davantage se focaliser sur certains enfants en plus grande difficulté pendant que d'autres travaillent avec l'outil d'aide pédagogique. Son objectif n'est pas de remplacer le rôle majeur de l'enseignante mais bien de permettre aux élèves de rester acteurs et de participer aux apprentissages scolaires de façon équitable. L'ensemble de ces facteurs a permis d'obtenir une intention d'usage de l'outil, et donc une bonne acceptabilité par les élèves. L'utilisabilité est un déterminant nécessaire, mais insuffisant pour l'assurance d'un usage effectif, l'appréciation subjective de la technologie par les utilisateurs joue un rôle important pour son acceptabilité [Dillon \& Morris 1999]. De plus, l'outil que nous avons développé répond aux sept principes du design universel :

- Une utilisation équitable pour tous. L'outil proposé ne présente pas de limitation selon le profil de l'enfant, le même usage est prévu pour tous quelles que soient les déficiences. Par exemple, l'utilisation d'une commande vocale permet à un élève aveugle ou avec des problèmes de motricité des bras d'utiliser l'outil.

- Une flexibilité et une souplesse d'usage à travers notamment la possibilité de paramétrer l'ensemble du contenu pédagogique et son accès par l'élève en fonction du niveau. Également, la tablette peut être utilisée selon différentes modalités : usage de la fonctionnalité tactile de l'écran, de la souris ou encore d'un trackball.

- Une manipulation simple et intuitive. Les élèves se sont rapidement appropriés l'outil, ils ne montrent pas de problème de navigation ou de compréhension des différentes actions possibles.

- Les informations sont perceptibles immédiatement et via différentes modalités (visuelle, auditive).

- Une tolérance à l'erreur notamment grâce aux feedbacks affichés aux élèves (modifications de couleurs de fond et messages fournissant un retour aux élèves suite à leurs actions et leur permettant de corriger les erreurs commises).

- Un faible niveau d'effort physique : la tablette est facilement transportable, légère et peut être utilisée avec ou sans clavier.

- Nous devons encore apporter des améliorations afin de répondre correctement au dernier principe concernant la dimension et l'utilisation de l'espace pour une bonne approche et utilisation. Nous souhaiterions, par exemple, mettre à disposition des élèves un écran de taille plus importante pour répondre de manière plus adaptée à leurs déficiences physiques.

L'intérêt de ce premier travail consistait à vérifier la faisabilité de l'intégration du numérique dans une classe auprès d'enfants en situation de multi-handicap ; les résultats sont très positifs. Pour la suite de cette étude, nous prévoyons d'inclure totalement l'outil dans la classe dès la rentrée de septembre 2014. Nous sommes en train d'intégrer l'ensemble de la méthode pédagogique mise en place par l'enseignante dans le cadre de l'apprentissage de la lecture, ainsi que d'autres contextes pédagogiques tels que les mathématiques, pour que l'outil soit complet et puisse être utilisé 
régulièrement et pendant une année scolaire complète. Il sera également testé auprès d'autres enfants de l'école. Il serait aussi pertinent de tester cette méthode auprès d'une autre population dite fragile, telle que les personnes âgées, et dans d'autres contextes, comme ceux des EHPAD.

L'objectif à plus long terme serait d'identifier la meilleure façon de diffuser cette méthode auprès de concepteurs et de les inciter à mettre en place et à développer la démarche de conception que nous proposons. L'idée serait que les concepteurs intègrent cette démarche afin que les contraintes maximales soient systématiquement prises en compte. Chacun pourrait alors bénéficier d'un grand panel d'outils et de services, sans nécessité d'ajustement particulier. Également, dans le cadre du développement de l'outil d'aide pédagogique, l'enseignante a souligné à plusieurs reprises que l'utilisation d'un tel outil serait vraiment pertinente dans un contexte pédagogique normal, pour aider les enfants en difficultés scolaires et certains élèves porteurs de handicaps intégrés.

\section{Bibliographie}

Bastien, J.M.C. \& Scapin, D.L., 1992. A validation of ergonomic criteria for the evaluation of human-computer interfaces. International Journal of Human-Computer Interaction, 4(2), pp. $183-196$.

DOI : $10.1080 / 10447319209526035$

Belio, C., 2012. Handicap, cognition et représentations.

Chabrol, B., 2006. Handicaps de l'enfant, Wolters Kluwer France.

Choi, S., 2005. Universal Design : A Practical Tool for a Diverse Future. International Journal of the Diversity, $\mathrm{N}^{\circ}$ 6, pp. 116-124.

Cremadeills, J. et al., 2002. Analyse de l'organisation et du fonctionnement des établissements régionaux d'enseignement adapté (EREA),

Dillon, a. \& Morris, M., 1999. Power, Perception and Performance : From Usability Engineering to Technology Acceptance with the P3 Model of User Response. Proceedings of the Human Factors and Ergonomics Society Annual Meeting, 43(19), pp. 1017-1021.

DOI : $10.1177 / 154193129904301901$

Lespinet-Najib, V. et al., 2013. "Design universel et Accessibilité : quels enjeux pour une société "nouvelle" ? In 4ème Colloque international "Jules Verne : sciences, crises et utopies."Nantes, Frnace.

Mace, R., Hardie, G.J. \& Place, J.P., 1991. Accessibility environments : Toward Universal Design, téléchargeable sur http://www.ncsu.edu/ncsu/design/cud/pubs_p/pud.htm.

Mayhew, D.J., 1999. The usability engineering lifecycle: A practitioner's handbook for user interface design, ACM.

Nielsen, J., 1994a. Usability inspection methods. In Wiley \& sons, ed. Conference companion on Human factors in computing systems. ACM, pp. 413-414.

Nielsen, J., 1994b. Usability inspection methods. In Wiley \& sons, ed. Conference companion on Human factors in computing systems. ACM, pp. 413-414.

Novak, J. \& Cañas, A., 2008. The theory underlying concept maps and how to construct and use them. Florida Institute for Human and Machine Cognition, pp. 1-36.

Organisation Internationale de Normalisation, 1999. ISO 13407: Human-Centered Design Processes for Interactives Systems. International Organization for Standardization.

\section{Table des illustrations}

Titre Figure 1. Interdépendance des différentes étapes de la CCU 
URL http://terminal.revues.org/docannexe/image/673/img-1.jpg

Fichier image/jpeg, 556k

Titre Figure 2. Étapes successives et itératives de la méthode de conception systémique

URL http://terminal.revues.org/docannexe/image/673/img-2.jpg

Fichier image/jpeg, 656k

\begin{tabular}{|c|c|c|}
\hline$=\underline{-}=$ & Titre & $\begin{array}{l}\text { Figure } 3 \text { : Représentation des éléments qui composent l'environnement } \\
\text { (logiciel XMind) }\end{array}$ \\
\hline E & URL & http://terminal.revues.org/docannexe/image/673/img-3.jpg \\
\hline & Fichier & image/jpeg, 148k \\
\hline & Titre & $\begin{array}{l}\text { Figure } 4 \text { : Exemple d'une situation d'action caractéristique et des } \\
\text { prérequis pour la réaliser }\end{array}$ \\
\hline 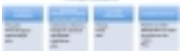 & URL & http://terminal.revues.org/docannexe/image/673/img-4.jpg \\
\hline & Fichier & image/jpeg, 568k \\
\hline 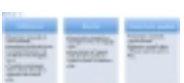 & Titre & $\begin{array}{l}\text { Figure } 5 \text { : Profils des trois élèves en termes de déficiences, besoins et } \\
\text { points forts }\end{array}$ \\
\hline 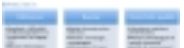 & URL & http://terminal.revues.org/docannexe/image/673/img-5.jpg \\
\hline & Fichier & image/jpeg, 168k \\
\hline 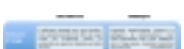 & Titre & Figure 6 : Description de trois méthodes d'utilisabilité \\
\hline 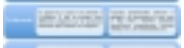 & URL & http://terminal.revues.org/docannexe/image/673/img-6.jpg \\
\hline 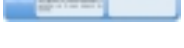 & Fichier & image/jpeg, 868k \\
\hline 夏 & Titre & Figure 7 : Illustrations du système d'aide pédagogique \\
\hline 国要国 & URL & http://terminal.revues.org/docannexe/image/673/img-7.jpg \\
\hline
\end{tabular}

\section{Pour citer cet article}

Référence électronique

Amélie Roche, Véronique Lespinet-Najib, Valérie Demanes, Isabelle Fermingier et Jean-Marc André, " Adaptation des TIC dans un contexte pédagogique pour des élèves en situation de multi-handicap », Terminal [En ligne], 116 | 2015, mis en ligne le 25 décembre 2014, consulté le 09 juillet 2017. URL : http://terminal.revues.org/673 ; DOI : 10.4000/terminal.673

\section{Auteurs}

\section{Amélie Roche}

Équipe CIH-IMS (UMR 5218), 109 avenue Roul, 33400 Talence. Tel. : 06.40.58.68.88 / Mail : amelie.roche@ensc.fr / Adresse postale : 16 rue du Soleil, 33000 Bordeaux.

\section{Véronique Lespinet-Najib}

Équipe ClH-IMS (UMR 5218), 109 avenue Roul, 33400 Talence.

Articles du même auteur

L'accessibilité Web, en 2013, en France : Enquête nationale sur les pratiques et les usages des professionnels du Web [Texte intégral]

Paru dans Terminal, 116 | 2015

Valérie Demanes

EREA/LEA LA Plaine, 28 rue du Moulineau, BP 75, 33326 Eysines cedex. 
Jean-Marc André

Équipe CIH-IMS (UMR 5218), 109 avenue Roul, 33400 Talence.

\section{Droits d'auteur}

tous droits réservés 